\title{
DESENVOLVIMENTO DE FITOCOSMÉTICO ANTIOXIDANTE COM EXTRATO DE LARANJA (Citrus sinensis)
}

\author{
GABRIELA WOORD MELLO GNANN, \\ CRISTIANE DE ALMEIDA, \\ DANIELE MICHELIN PAGANOTTE, \\ VERA ISAAC, \\ BRUNA GALDORFINI CHIARI-ANDRÉO, \\ PAULA CRESSONI MARTINI-MARCIANO.
}

\begin{abstract}
RESUMO - Orange (Citrus sinensis) is one of the most cultivated fruits in Brazil. It is known that it contains a relevant amount of vitamin $\mathrm{C}$ and, consequently, many properties, such as antioxidant, vasoprotective and hypocholesterolemic. The increase in the life expectancy stimulates the use of products able to retard the effects of time specially in the skin and, in this context, the use of plants and, particularly, of their extracts, came to revolutionize the modern cosmetology. Substances able to inhibit the action of free radicals, such as the vitamin $\mathrm{C}$, can be used in anti-aging cosmetics helping in the prevention or deceleration of the damage produced on the skin. Therefore, this study aimed to evaluate the viability in use orange as a source of antioxidants to the cosmetic industry, mainly for the prepare of antianging formulations. Thus, three orange extracts using different extractive processes were prepared followed by the evaluation of their antioxidant activity. A phytocosmetic was developed with the orange extract that exhibited the higher antioxidant activity, and it was characterized in relation to its organoleptical characteristics, $\mathrm{pH}$, viscosisity and rheology. Also, the microbiological aspects of the phytocosmetic was assessed. The extract prepared by the maceration of the dried orange, using $70 \%$ ethanol as solvent, showed significant antioxidant activity, thus, it was used in the phytocosmetic's development. The pH, viscosity, rheology and organoleptic characteristics determined is suitable to the use in the skin. The microbiological evaluation demonstrated the suitability of the formulation, according to the recommendations of the Brazilian Pharmacopoeia (2019). In conclusion, were demonstrated the applicability of orange extracts in antioxidant phytocosmetics, which could help in the prevention of premature skin aging using a widely available fruit in Brazil.
\end{abstract}

PALAVRAS-CHAVE - Antioxidant; Citrus sp extract; Phytocosmetic.

\section{INTRODUÇÃO}

Antioxidantes são substâncias que retardam a velocidade da oxidação de determinadas substâncias e substratos biológicos, por meio de um ou mais mecanismos como, por exemplo, inibição de radicais livres e são encontrados em diversas plantas ricas em compostos fenólicos, flavonoides, entre outros [1].

São substâncias extremamente utilizadas na atualidade devido a estas propriedades, considerando a intenção de muitas pessoas em retardar os efeitos do tempo sobre o corpo, principalmente sobre a pele. Este comportamento torna-se mais frequente e extremado com o aumento da expectativa de vida [2], [3].

Nesta busca por produtos antienvelhecimento, com atividade antioxidante, o emprego das plantas e, particularmente, dos seus extratos, tem revolucionado a cosmética moderna, pois o uso de produtos sintéticos criou, muitas vezes, situações toxicológicas graves [4].

Substâncias capazes de inibir a ação dos radicais livres, como a vitamina $\mathrm{C}$, encontrada na composição de diversas frutas, podem ser empregadas em cosméticos antienvelhecimento, auxiliando na prevenção ou desaceleração dos danos causados à pele [5]-[7].

Pertencente à família das Rutáceas (Rutaceae), a laranja (Citrus sinensis) é uma das frutas mais cultivadas no Brasil e popularmente conhecida pela quantidade relevante de vitamina $\mathrm{C}$, em sua composição [8].

É sabido que as frutas cítricas são cultivadas em todo o mundo, sendo que o Brasil se destaca juntamente com outros países produtores, como Índia, Espanha, África do Sul, entre outros [9]. Trata-se de uma fruta já empregada no setor cosmético para a extração do óleo essencial de sua casca, 
contudo, há necessidade de estudos sobre a possibilidade de emprego de todas as suas partes neste setor [10].

Assim sendo, o presente trabalho teve como objetivo avaliar a viabilidade da utilização da laranja (Citrus sp.) para o preparo de um cosmético antienvelhecimento. Desta forma, diferentes métodos de extração foram testados, permitindo a verificação de qual oferece o melhor potencial antioxidante e características microbiológicas aceitáveis de acordo com os parâmetros preconizados pela Farmacopeia Brasileira (2019) [11].

\section{METODOLOGIA}

\section{Material vegetal}

As laranjas (Citrus sinensis) frescas e maduras foram coletadas na Fazenda Itaqui, localizada no município de Estiva Gerbi - SP. Durante a coleta, os frutos selecionados foram separados de frutos lesionados e de materiais estranhos.

\section{Preparo dos extratos}

Para o preparo dos extratos, as laranjas selecionadas foram lavadas com água corrente, seguida de desinfecção em solução de hipoclorito de sódio a $0,1 \%$, por 20 minutos, e posterior lavagem com água destilada.

Em seguida, foram preparados três extratos empregando os seguintes processos extrativos:

- Processo extrativo 1: A solução extrativa da laranja foi obtida com auxílio de um espremedor de frutas doméstico, obtendo-se o extrato 1.

- Processo extrativo 2: A solução extrativa da laranja foi obtida com auxílio de um espremedor de frutas doméstico. Em seguida o suco foi filtrado e rotaevaporado até a eliminação da fase aquosa à temperatura máxima de $65^{\circ} \mathrm{C}$, obtendo-se o extrato 2.

- Processo extrativo 3: As frutas, juntamente com as cascas, foram cortadas em pequenos pedaços, acondicionados em estufa de ar circulante a $45^{\circ} \mathrm{C}$, por 72 horas, para desidratação. As laranjas secas foram, então, trituradas em liquidificador industrial. Este material foi macerado por 120 horas com etanol $70 \%$, na proporção $1: 1(\mathrm{~m} / \mathrm{v})$. Este procedimento foi realizado ao abrigo da luz, evitando a possível degradação de componentes fotossensíveis, como a vitamina $\mathrm{C}$. Ao final da maceração, a solução extrativa foi filtrada e o resíduo sólido foi descartado. O filtrado foi rotaevaporado a $60^{\circ} \mathrm{C}$, para a remoção do etanol. A fração aquosa do extrato obtido foi armazenada em frasco de vidro âmbar e acondicionada, sob refrigeração, até o momento do uso. Este extrato foi denominado como extrato 3.

Avaliação da atividade antioxidante dos extratos

A avaliação da atividade antioxidante dos extratos de laranja foi determinada de acordo com a metodologia descrita por Sanchez-Moreno (1998) [12].

Um mililitro de diferentes concentrações do extrato de laranja solubilizado em $9 \mathrm{~mL}$ água foram pipetados em tubos de ensaio. A estes tubos, foram adicionados $2 \mathrm{~mL}$ da solução de radical DPPH• (2,2 difenil-1-picril hidrazil) a 0,04\%. As soluções foram incubadas, por 30 minutos, na ausência de luz e, em seguida, foi realizada a leitura em espectrofotômetro, a $517 \mathrm{~nm}$. Este ensaio foi realizado em triplicata.

Um branco foi preparado da mesma forma que a amostra, utilizando $1 \mathrm{~mL}$ de água destilada em substituição aos extratos. Foi possível calcular a porcentagem de inibição do radical DPPH causada por cada uma das diluições do extrato de laranja de acordo com a Equação 1 (Figura 1).

$$
\% \text { de inibição }=\frac{\text { Amáx }- \text { Aamostra }}{\text { Amáx }} \times 100 \text { (Equação 1) }
$$

Figura 1. Equação 1

Na Equação 1 (Figura 1), Amáx corresponde à absorbância do branco, portanto, na ausência de extrato e Aamostra corresponde à absorbância das amostras, ou seja, as soluções contendo os extratos da laranja.

Com estes valores, foram construídas curvas dose $\mathrm{x}$ resposta, ou seja, as concentrações do extrato de laranja versus a porcentagem de inibição do radical DPPH promovida por cada uma das concentrações testadas. Esta prática permitiu a determinação das equações matemáticas que descrevem estas curvas e, em seguida, verificar o valor do IC50 dos extratos estudados. O IC50 é descrito como a concentração destas substâncias capaz de inibir 50\% dos radicais DPPH em solução.

Para a determinação da eficiência deste método, o mesmo ensaio foi repetido utilizando uma solução de vitamina $\mathrm{C}$ (padrão antioxidante). Para isto, a vitamina $\mathrm{C}$ foi diluída a partir de uma solução mãe a $200 \mu \mathrm{g} / \mathrm{mL}$ nas concentrações $10,12,14,16,18$ e $20 \mu \mathrm{g} / \mathrm{mL}$.

$\mathrm{O}$ extrato que apresentou melhor atividade antioxidante foi selecionado para o desenvolvimento do fitocosmético, bem como para a realização da continuidade dos ensaios descritos a seguir.

\section{Avaliação fitoquímica do extrato}

$\mathrm{O}$ extrato de melhor desempenho na avaliação da atividade antioxidante foi submetido também às avaliações fitoquímicas através de testes clássicos de identificação para detecção de flavonoides e taninos [13].

Para a caracterização dos flavonoides realizaram-se os testes clássicos de identificação: reação de Shinoda, reação de cloreto férrico e reação de cloreto de alumínio. Para a caracterização dos taninos realizaram-se os testes clássicos de identificação: reação com acetato de chumbo e reação com sais de ferro.

\section{Desenvolvimento do fitocosmético}

Foi desenvolvida uma emulsão óleo-em-água não-iônica, utilizando os conservantes usualmente empregados no Brasil, adequados para bases e ativos cosméticos.

A formulação foi preparada de forma convencional, aquecendo-se a fase aquosa e oleosa em béqueres separados, até a solubilização e/ou fusão dos componentes. Em seguida, a fase aquosa foi vertida na oleosa e o sistema mantido em agitação constante até o resfriamento. Após resfriamento da 
formulação, o extrato (5\%) que apresentou melhor atividade antioxidante foi incorporado na emulsão para obtenção do fitocosmético.

Para obter indícios sobre a estabilidade deste fitocosmético, a formulação foi acompanhada por um período de 15 dias em diferentes condições de temperatura. Uma amostra foi mantida à temperatura ambiente, porém ao abrigo da luz; outra amostra foi exposta à radiação luminosa, sendo envasada em frasco de vidro transparente. Ainda, houve amostras submetidas a diferentes temperaturas, entre elas: $5{ }^{\circ} \mathrm{C}$ (armazenada em geladeira), $-5{ }^{\circ} \mathrm{C}$ (armazenada em freezer), $45^{\circ} \mathrm{C}$ (sendo armazenada em estufa) e ciclos de congelamento e descongelamento ( $24 \mathrm{~h}$ na estufa e $24 \mathrm{~h}$ no freezer por 12 dias). Estas diferentes temperaturas e condições podem ser entendidas como situações de estresse, por forçarem o aparecimento de instabilidades na formulação.

Os caracteres organolépticos, como aspecto, cor e odor foram avaliados diariamente. Ainda, o pH de uma dispersão (a $10 \%$ em água purificada) do fitocosmético e sua viscosidade foram medidos, em triplicata. Todos estes são parâmetros recomendados pelo Guia de Estabilidade de Produtos Cosméticos da ANVISA [14].

A determinação do $\mathrm{pH}$ foi realizada com auxílio de peagômetro digital. A viscosidade das formulações durante a estabilidade foi verificada com o auxílio de um reômetro Haake RS-1. Neste tipo de equipamento são realizados ensaios dinâmicos, desta forma, não é possível a determinação de um valor absoluto da viscosidade das formulações. Por este motivo, o parâmetro utilizado foi à viscosidade aparente mínima, que é a viscosidade apresentada pelas formulações quando submetidas a uma tensão de cisalhamento máximo (100 s-1) no ensaio da curva de fluxo.

\section{Caracterização reológica}

O fitocosmético teve seu comportamento reológico avaliado em reômetro HAAKE modelo RHEOSTRESS RS1 , mesmo equipamento utilizado para determinação da viscosidade aparente, utilizando o sensor do tipo cone-placa $\left(\mathrm{C} 35 / 2^{\circ} \mathrm{Ti}\right)$ e os dados analisados pelo software Rheowin 3. Analisou-se o comportamento reológico da emulsão, determinando-se: (1) Propriedades de fluxo das amostras, a tixotropia e a área de histerese, com tensão de cisalhamento de 0 -100 Pa por um período de 120 segundos para curva ascendente e 120 segundos para a curva descendente, obtendo dados a cada segundo; (2) Varredura de tensão, com tensão de cisalhamento de 0,01-40 Pa, por 30 segundos numa frequência de $1 \mathrm{~Hz}$; (3) Varredura de frequência, submetendo as amostras a uma tensão de cisalhamento de $2 \mathrm{~Pa}$, utilizando frequências variáveis de $5.10-3 \mathrm{~Hz}$ a $35 \mathrm{~Hz}$; (5) Curva de fluência e relaxação com tensão de cisalhamento de $0,5 \mathrm{~Pa}$ por 30 minutos. Todos os ensaios foram realizados a $32{ }^{\circ} \mathrm{C}$ e em triplicata [15].

\section{Avaliação microbiológica}

As análises microbiológicas foram realizadas com o extrato de melhor desempenho como antioxidante e com o fitocosmético desenvolvido. Para ambos, as amostras foram avaliadas após sete dias do preparo.
Foi empregada a metodologia preconizada pela Farmacopéia Brasileira (2019) [11] para a pesquisa de microorganismos viáveis e patógenos (Salmonella sp., Escherichia coli, Pseudomonas aeruginosa, Sthaphylococcus aureus), utilizando técnicas assépticas, em capela de fluxo laminar.

A contagem total de micro-organismos mesófilos foi realizada com a diluição das amostras em tampão fosfato $(\mathrm{pH}$ 7,2 , estéril), na proporção de 1:10. No caso do fitocosmético, após diluição, foi realizada a adição de $0,1 \mathrm{~mL}$ de polisorbato 80 para inativação do sistema conservante, constituído de parabenos.

\section{RESULTADOS E DISCUSSÃO \\ Preparação do extrato}

Os extratos da laranja foram preparados a partir dos cultivares de Citrus sinensis de acordo com os processos extrativos especificados na metodologia. O objetivo do emprego de diferentes métodos extrativos consistiu em verificar se, o potencial antioxidante, já descrito para a laranja, mantém-se na extração por métodos que poderiam ser empregados no setor cosmético, inclusive com solventes não agressores ao meio ambiente e aos usuários, como é o caso da água e do etanol.

\section{Avaliação antioxidante dos extratos de laranja}

A avaliação da atividade antioxidante dos extratos foi realizada utilizando-se o método do radical DPPH•, extensivamente aplicado para o estudo de potenciais ativos cosméticos [16], [17].

Este resultado foi utilizado como parâmetro para descrever o método mais adequado, entre os três testados, para a extração dos componentes de interesse da laranja, quando se considera o interesse cosmético.

Além disso, foi útil para a escolha de qual o extrato deveria ser usado na preparação do fitocosmético desenvolvido neste estudo, já que o intuito principal deste produto é que apresente atividade contra radicais livres produzidos na pele, por fatores intrínsecos (metabolismo celular) e extrínsecos (poluição, fumo, radiação ultravioleta, entre outros).

Neste momento, é válido ressaltar que o emprego na laranja no setor cosmético não é uma novidade, considerandose o emprego como fonte de óleos essenciais, principalmente para a preparação de fragrâncias [18].

Também, Wuttisin et al. (2017) [19] testou o uso da casca para um cosmético clareador, avaliando sua capacidade de inibir a atividade da enzima tirosinase. Ainda, uma patente [20] descreve o emprego das folhas, flores e casca para o uso cosmético, mais especificamente antioxidante.

Entretanto, a descrição da utilização da fruta como fonte de ativos antioxidantes para este setor, não foi encontrada na literatura por nosso grupo de pesquisa.

As curvas dose $\mathrm{x}$ resposta construídas com os resultados obtidos estão apresentadas nas Figuras 2 a 4.

As Figuras 2 a 4 indicam que, mesmo as menores concentrações testadas neste estudo, foram suficientes para exibir capacidade antioxidante. Mas, para que seja possível uma melhor comparação destes resultados com outras frutas, bem 


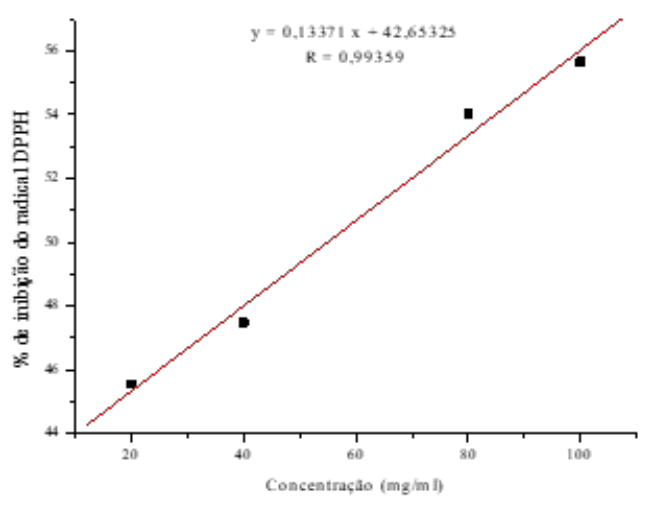

Figura 2. Curva dose x resposta, indicando porcentagem de inibição promovida ao radical DPPH• por diferentes concentrações do extrato 1.

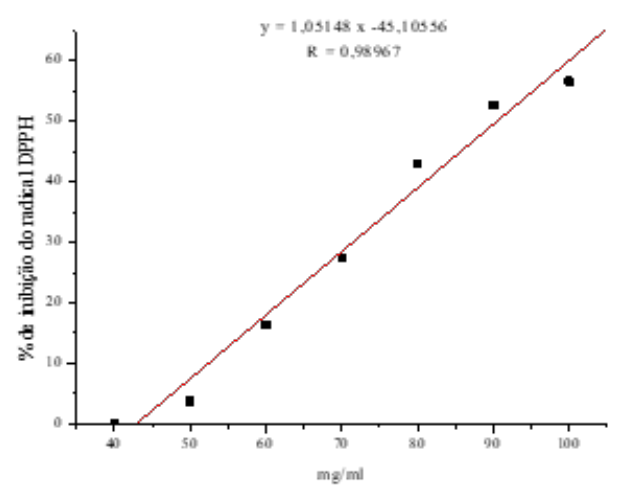

Figura 3. Curva dose x resposta, indicando porcentagem de inibição promovida ao radical DPPH• por diferentes concentrações do extrato 2 .

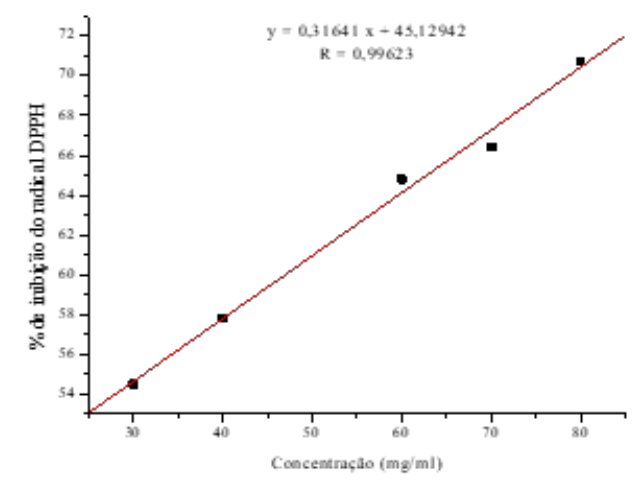

Figura 4. Curva dose $x$ resposta, indicando porcentagem de inibição promovida ao radical DPPH• por diferentes concentrações do extrato 3.

como entre os diferentes métodos de extração avaliados, é conveniente a avaliação numérica por meio do $\mathrm{IC}_{50}$.

A Tabela 1 mostra os valores de $\mathrm{IC}_{50}$ calculados para cada um dos extratos, obtido por meio da regressão linear das curvas.

As reações de Shinoda, para verificação da presença de flavonoides, e com acetato de chumbo, para confirmação da presença de taninos, apresentaram resultados positivos, caracterizando a presença destes dois grupos de metabólitos secundários no extrato obtido, sendo, provavelmente,
Tabela 1. Caracterização fitoquímica do extrato de laranja.

\begin{tabular}{|l|l|}
\hline Reação & Resultados \\
\hline Reação de Shinoda & + \\
\hline Reação de cloreto férrico & - \\
\hline Reação de cloreto de alumínio & - \\
\hline Reação com sais de ferro & - \\
\hline Reação com acetato de chumbo & + \\
\hline
\end{tabular}

responsáveis pelo poder antioxidante exibido pelo extrato, juntamente com a vitamina $\mathrm{C}$ [13].

\section{Desenvolvimento do fitocosmético}

$\mathrm{Na}$ Tabela 2 encontra-se a composição percentual da formulação desenvolvida para incorporação do extrato 3. Tratase de um protótipo, com a intenção de comprovar a possibilidade de incorporação do extrato obtido para a finalidade antienvelhecimento.

Tabela 2. Composição percentual da formulação desenvolvida para a adição do extrato 3 (Denominação das matérias-primas de acordo com a International Nomenclature of Cosmetic Ingredients - INCI).

\begin{tabular}{|l|c|}
\hline Componentes & Composição percentual (\% m/m) \\
\hline Ceteareth-20 & 3,00 \\
\hline Cetearyl Alcohol & 3,00 \\
\hline Isopropyl Palmitate & 2,50 \\
\hline Glyceryl stearate & 4,00 \\
\hline Disodium EDTA & 0,05 \\
\hline Metylparaben & 0,18 \\
\hline Propilparaben & 0,02 \\
\hline Propylene Glycol & 3,00 \\
\hline Aqua & q.s.p. 100,00 \\
\hline
\end{tabular}

O extrato de Citrus sativum foi acrescentado à formulação após seu resfriamento na concentração de 5\% para a obtenção do fitocosmético, caracterização desta formulação e avaliação microbiológica. Não houve necessidade de solubilização prévia do extrato, pois, por se tratar de um extrato mole, a incorporação foi facilitada.

Esta concentração (5\%) baseia-se no resultado do ensaio de atividade antioxidante, em que, fazendo uma correlação simples, a cada $1 \mathrm{~g}$ de formulação, haveria a necessidade de $15,39 \mathrm{mg}$ do extrato para atingir o IC50; portanto, a concentração estimada seria de $1,5 \%$. Considerando que a penetração do extrato na pele, por meio da aplicação tópica, não é de $100 \%$, a concentração empregada corresponde a, aproximadamente, 3 vezes a concentração efetiva, conforme dados da literatura [23]. Obviamente, outros ensaios, como o de liberação e permeação cutânea podem ser realizados para melhor compreensão do comportamento da formulação ao ser aplicada na pele.

Quanto ao acompanhamento dos caracteres organolépticos do fitocosmético, pode-se inferir que o cosmético desenvolvido se apresentou como uma formulação de aspecto homogêneo, coloração amarela clara e odor característico. Durante 15 dias de análise, a formulação permaneceu sem 
alterações em relação ao aspecto, cor e odor, mesmo quando submetida às diferentes condições de estresse. Não ocorreu separação de fases, precipitação ou turvação.

$\mathrm{O} \mathrm{pH}$ também foi avaliado, pois pode indicar hidrólise ou outras alterações que podem ocorrer devido ao tempo de estocagem e/ou condições inadequadas de transporte e armazenamento, até mesmo contaminações [24].

Os valores de $\mathrm{pH}$ medidos estão de acordo com o preconizado para produtos de aplicação na pele, apresentando valores compatíveis com o pH cutâneo de 5,5 a 6,5. A Figura 5 apresenta os valores de $\mathrm{pH}$ determinados da estabilidade preliminar com o desvio padrão e a variância das médias dos valores de $\mathrm{pH}$.

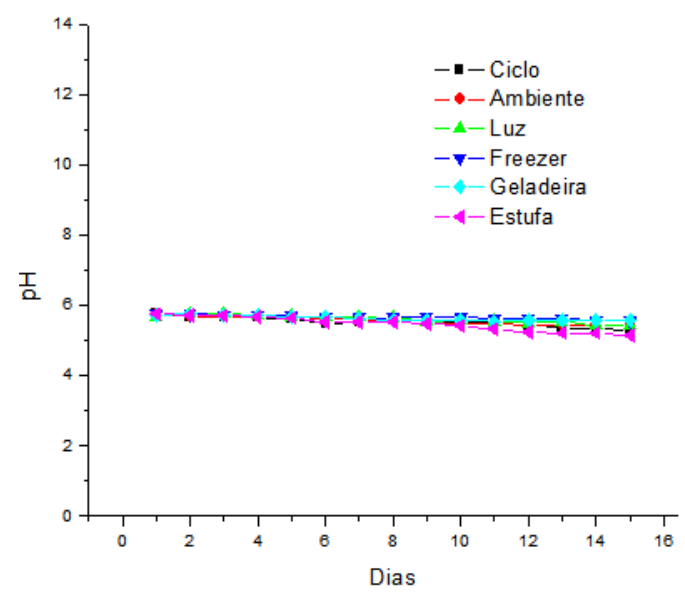

Figura 5. Valores de $\mathrm{pH}$ determinados durante 15 dias de estudo do fitocosmético.

No estudo da viscosidade, mostrado na Figura 6, nota-se redução da viscosidade das formulações com o passar do tempo, com exceção da formulação armazenada a $45^{\circ} \mathrm{C}$, que apresentou aumento na viscosidade. Isto pode ser devido a evaporação da água contida no creme, devido à elevada temperatura a que foi submetida. Com a evaporação da água, a maior concentração de componentes graxos, torna a emulsão mais viscosa, não sendo isto, um sinal de instabilidade.

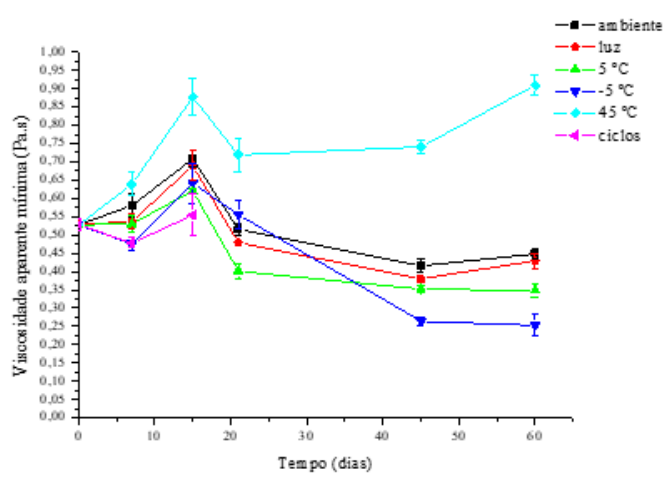

Figura 6. Valores de viscosidade determinados para o fitocosmético durante 60 dias após a manipulação.

Por ter sido a viscosidade um parâmetro com variação significativa nos primeiros dias após a manipulação, optouse por acompanhar este parâmetro por um período maior, portanto, 60 dias.

\section{Caracterização reológica}

De acordo com a análise da curva de fluxo (Figura 7), a formulação pode ser considerada como um fluido nãoNewtoniano. Além disso, devido à área de histerese presente entre as curvas de subida e descida, é caracterizada também como um fluido tixotrópico.

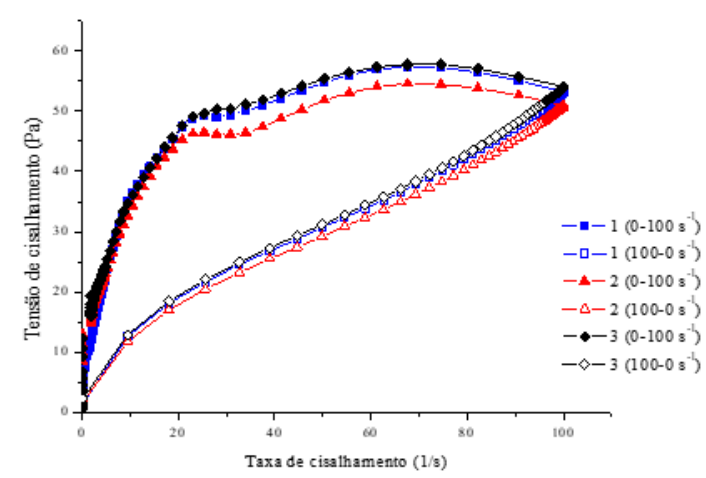

Figura 7. Curva de fluxo do fitocosmético.

Estes parâmetros são interessantes para uma formulação cosmética, considerando que a redução da viscosidade com o cisalhamento torna a aplicação mais fácil para o usuário e, além disso, a presença da área de histerese indica facilidade de liberação das substâncias ativas contidas na formulação [25].

A análise da varredura de tensão (Figura 8) é útil na determinação da faixa de tensão onde se observa um comportamento viscoelástico linear, ou seja, uma faixa de tensão em que a formulação flui, mas que não sofre deformações estruturais irreversíveis. Neste ensaio, foi determinado que a tensão de cisalhamento adequada para a realização dos ensaios de fluência e relaxação e varredura de frequência, é de $1 \mathrm{~Pa}$ [15].

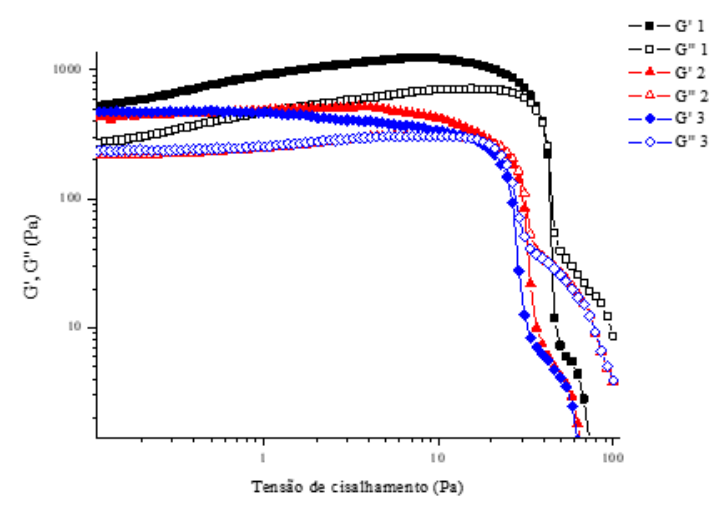

Figura 8. Varredura de tensão do fitocosmético.

A varredura de frequência (Figura 9) também demonstra 
o comportamento viscoelástico da formulação, apresentando G' maior que G', ou seja, módulo elástico maior que o módulo viscoso.

Este comportamento é muito importante em formulações cosméticas, principalmente em emulsões, pois pode significar uma maior estabilidade da formulação [25].

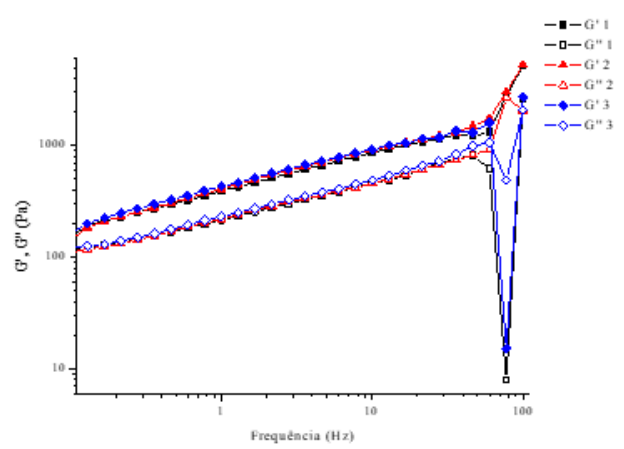

Figura 9. Varredura de frequência do fitocosmético.

O ensaio de fluência e relaxação (Figura 10) mostra o comportamento viscoelástico da formulação, que se deforma quando submetida à uma tensão de cisalhamento, assim como uma fluido viscoso, mas recupera sua estrutura quando cessa o cisalhamento, como um fluido elástico.

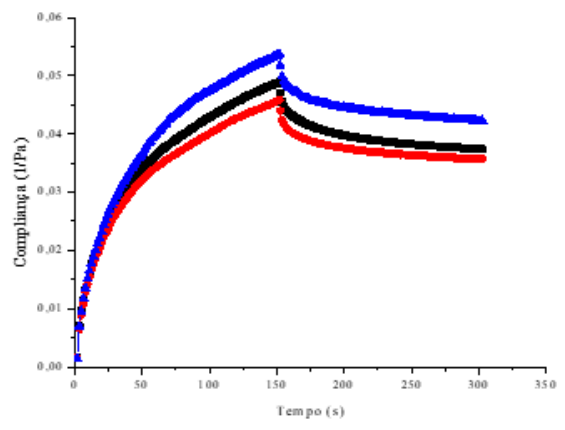

Figura 10. Ensaio de fluência e relaxação do fitocosmético.

\section{Controle de qualidade microbiológico}

A Tabela 3 mostra o resultado da avaliação microbiológica conduzida para o extrato 3 e para o fitocosmético.

As especificações descritas na Tabela 3 estão de acordo com a Farmacopeia Brasileira (2019) [11]. Este dado, permite inferir, portanto, que o método de extração empregado não interfere negativamente na qualidade microbiológica do extrato obtido. Também, o fitocosmético preparado com o Extrato 3 atende aos parâmetros microbiológicos preconizados.

\section{CONCLUSÕES}

Pelos resultados foi possível verificar que a extração, por maceração, utilizando solução hidroetanólica a 70\%, resultou em um extrato com componentes que apresentaram maior capacidade de sequestrar radicais livres; ou seja, maior atividade antioxidante. Dentre os métodos avaliados, é o que se recomenda, portanto, para o emprego no setor cosmético.
Desta forma, os resultados indicam que, para a extração seletiva de antioxidantes naturais, é de grande importância o estudo sobre o solvente e o método de extração apropriado.

Além disso, comprovou-se a viabilidade em empregar a laranja como fruta capaz de oferecer compostos antioxidantes para a preparação de um fitocosmético antienvelhecimento, inclusive com parâmetros físico-químicos e microbiológicos aceitáveis. Este é um dado interessante, considerando a grande disponibilidade da fruta no território brasileiro. Tratase de mais uma forma de agregar valor a este cultivar tão disseminado no Brasil.

\section{Referências}

[1] C. A. Rice-Evans, N. J. Miller, G. Paganga. Structure-antioxidant activity relationships of flavonoids and phenolic acids. Free Radical Biology and Medicine, 20(7):933-956, 1996.

[2] L. Scotti, M. T. Scotti, C. Cardoso, P. Pauletti, I. Castro-Gamboa, V. S. Bolzani. Modelagem molecular aplicada ao desenvolvimento de moléculas com atividade antioxidante visando ao uso cosmético. Revista Brasileira de Ciências Farmacêuticas, 43(2):153-166, 2007.

[3] A. P. Teston, D. Nardino, L. Pivato. Envelhecimento cutâneo: teoria dos radicais livres e tratamentos visando a prevenção e o rejuvenescimento. Revista Uningá, 24(1):71-84, 2010.

[4] E. M. Kuskoski, A. G. Asuero, M. T. Morales, R. Fett. Frutos tropicais silvestres e polpas de frutas congeladas: atividade antioxidante, polifenóis e antocianinas. Ciência Rural, 36(4):1283-1287, 2006.

[5] P. U. Giacomini. Understanding reactive oxygen species. Cosmetics Toiletries, 122(5):36-40, 2007

[6] K. E. Burke. Interaction of vitamins $\mathrm{C}$ and $\mathrm{E}$ as better cosmeceuticals. Dermatologic Therapy, 20(5):314-321, 2007.

[7] H. Masaki. Role of antioxidants in the skin: anti-aging effects. Journal of Dermatological Science, 58(2):85-90, 2010

[8] E. I. M. Araujo, L. C. C. F. Monteiro, A. M. S. de Oliveira, L. A. Alves, L. M. Bertini. Caracterização da atividade antioxidante, teor de fenóis totais e atividade larvicida frente ao Aedes aegypti de Citrus sinensis L. (laranja). Blucher Chemistry Proceedings, 3(1), 2015. Disponível em: http://pdf.blucher.com.br.s3-sa-east1.amazonaws.com/chemistryproceedings/5erq4enq/org3.pdf.

[9] A. A. Sanofer. Role of citrus fruits in health. Journal of Pharmaceutical Sciences and Research, 6(2):121-123, 2014.

[10] D.C. Sikdar, R. Menon, K. Duseja, P. Kumar, P. Swami. Extraction of citrus oil from orange (Citrus sinensis) peels by steam distillation and its characterizations. International Journal of Technical Research and Applications, 4(3):341-346, 2016.

[11] Farmacopeia Brasileira. 6 ed, volume 1. São Paulo: ANVISA, 2019.

[12] C. Sánchez-Moreno, J. A. Larrauri, F. Saura-Calixto. A procedure to measure the antiradical efficiency of polyphenols. Journal of the Science of Food and Agriculture, 76(2):270-276, 1998.

[13] M. B. Falkenberg, S. R. I. Santo, C. M. O. Simões. Introdução à análise fitoquímica. In: Simões et al. (Org). Farmacognosia da planta ao medicamento, 6 a ed. UFRGS, 2007.

[14] Brasil. Agência Nacional de Vigilância Sanitária. Guia de estabilidade de produtos cosméticos. Brasília: Núcleo de Assessoramento em Comunicação Social e Institucional. 2004; 1: 12-19, 26, 27.

[15] V. L. B. Isaac, J. D. D. Moraes, B. G. Chiari, D. A. S. Guglielmi, L. C. Cefali, N. C. Rissi, M. A. Corrêa Determination of the real influence of the addition of four thickening agents in creams using rheological measurements, Journal of Dispersion Science and Technology, 34(4):532538, 2013.

[16] B. G. Chiari, J. A. Severi, P. A. D. Pauli-Credendio, C. M. D. Sylos, W. Vilegas, M. A. Corrêa, V. L. B. ISAAC. Assessment of the chemical profile, polyphenol content and antioxidant activity in extracts of Psidium guajava L. fruits. International Journal of Pharmacy and Pharmaceutical Sciences, 4(5):331-336, 2012.

[17] C. Tsai, L. Lin. DPPH scavenging capacity of extracts from Camellia seed dregs using polyol compounds as solventes. Heliyon, 5(8):e02315, 2019.

[18] C. L. Burnett, M. M. Fiume, W. F. Bergfeld, D. V. Belsito, R. A. Hill, C D. Klaassen, D. C. Liebler, J. G. Marks Jr, R. C. Shank, T. J. Slaga, P. W. Snyder, L. J. Gill, B. Heldreth. Safety assessment of citrus-derived peel 
Tabela 3. Resultado da avaliação microbiológica do extrato 3 e do fitocosmético.

\begin{tabular}{|l|l|l|l|l|}
\hline $\begin{array}{l}\text { Micro-organismo } \\
\text { avaliado }\end{array}$ & $\begin{array}{l}\text { Resultado obtido para } \\
\text { o extrato }(\mathbf{U F C} / \mathbf{m L})\end{array}$ & $\begin{array}{l}\text { Especificação } \\
\text { Extrato }(\mathbf{U F C} / \mathbf{m L}) *\end{array}$ & $\begin{array}{l}\text { Resultado obtido para } \\
\text { o fitocosmético (UFC/g) }\end{array}$ & $\begin{array}{l}\text { Especificação } \\
\text { Fitocosmético (UFC/g)** }\end{array}$ \\
\hline Bactérias & $<10^{2}$ & $10^{4}$ & $<10$ & $10^{2}$ \\
\hline $\begin{array}{l}\text { Fungos/ } \\
\text { Leveduras }\end{array}$ & $<10^{2}$ & $10^{2}$ & $<10$ & $10^{1}$ \\
\hline Patógenos & Ausente & Ausente & Ausente & Ausente \\
\hline
\end{tabular}

oils as used in cosmetics. International Journal of Toxicology, 38(2):33S59S, 2019.

[19] N. Wuttisin, J. Boonmak, V. Thaipitak, N. Thitilertdecha, K. Kittigowittana. Anti-tyrosinase activity of orange peel extract and cosmetic formulation. International Food Research Journal, 24(5):2128-2132, 2017.

[20] Patente US5916576A. Disponível em: https://patents.google.com/patent/US5916576A/en. Acesso em março de 2020.

[21] L. C. Cefali, J. A. Ataide, I. M. O. Sousa, M. C. Figueiredo, A. L. T. G. Ruiz, M. A. Foglio, P. G. Mazzola. In vitro solar protection factor, antioxidant activity, and stability of a topical formulation containing Benitaka grape (Vitis vinifera L.) peel extract. Natural Product Research, 12:1-6, 2019.

[22] B. G. Chiari-Andréo, E. Trovatti, J. Marto, M. G. J. Almeida-Cincotto, A. Melero, M. A. Corrêa, L. A. Chiavacci, H. Ribeiro, T. Garrigues, V. L. B. Isaac. Guava: phytochemical composition of a potential source of antioxidants for cosmetic and/or dermatological applications. Brazilian Journal of Pharmaceutical Sciences, 53(2):e16141, 2017.

[23] F. G. Leite, J. A. Oshiro Junior, L. A. Chiavacci, B. G. Chiari-Andréo Assessment of an anti-ageing structured cosmetic formulation containing goji berry. Brazilian Journal of Pharmaceutical Sciences, 55:e17412, 2019.

[24] V. L. B. Isaac, L. C. Cefali, B. G. Chiari, C. C. L. G. Oliveira, H. R. N. Salgado, M. A. Corrêa. Protocolo para ensaios físico-químico de estabilidade de fitocosméticos. Revista de Ciências Farmacêuticas Básica e Aplicada, 29(1):81-96, 2008.

[25] V. L. B. Isaac, B. G. Chiari-Andréo, J. M. Marto, J. D. D. Moraes, B. A. Leone, M. A .Corrêa, H. M. Ribeiro. Rheology as a tool to predict the release of alpha-lipoic acid from emulsions used for the prevention of skin aging. BioMed Research International, 2015:1-8, 2015

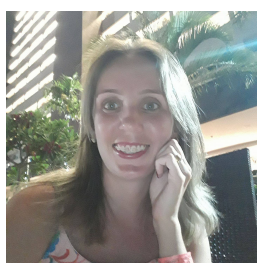

\section{GABRIELA WOORD MELLO GNANN}

Centro Universitário da Fundação Hermínio Ometto FHO - UNIARARAS, Av. Dr. Maximiliano Baruto, 500, 13607-339, Araras, Brasil.

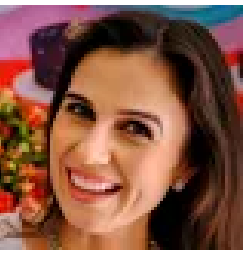

DANIELE MICHELIN PAGANOTTE

Centro Universitário da Fundação Hermínio Ometto FHO - UNIARARAS, Av. Dr. Maximiliano Baruto, 500, 13607-339, Araras, Brasil.

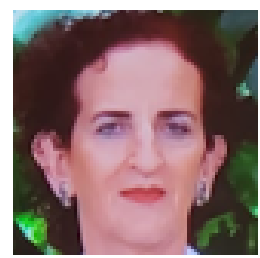

VERA ISAAC

Universidade Estadual Paulista (UNESP), Faculdade de Ciências Farmacêuticas, Araraquara LaCos, Rodovia Araraquara-Jaú, km-1, 14801-902, Brasil.

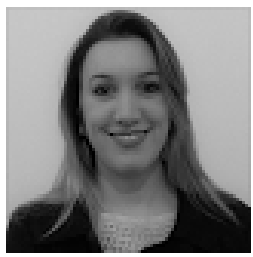

BRUNA GALDORFINI CHIARI-ANDRÉO Universidade de Araraquara-UNIARA, Rua Carlos Gomes, 1338, 14801-340, Araraquara, Brasil.

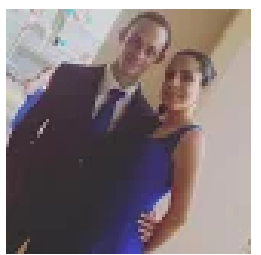

\section{CRISTIANE DE ALMEIDA}

Centro Universitário da Fundação Hermínio Ometto FHO - UNIARARAS, Av. Dr. Maximiliano Baruto, 500, 13607-339, Araras, Brasil.

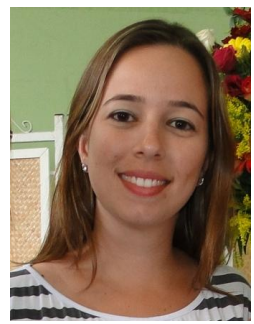

PAULA CRESSONI MARTINI-MARCIANO

Centro Universitário da Fundação Hermínio Ometto FHO - UNIARARAS, Av. Dr. Maximiliano Baruto, 500, 13607-339, Araras, Brasil e Universidade Paulista - UNIP, Rua Miguel Guidote, 405 - Parque Egisto Ragazzo, Limeira - SP, 13485342. 